\title{
Enfermedad de cambios mínimos cortico-resistente e hipotiroidismo. Reporte de caso
}

\author{
Steroid-resistant minimal change disease and hypothyroidism: Case report
}

José Leonel Zambrano-Urbano1* orcid.org/0000-0002-4959-9136

Darío Sebastián López Delgado²,3 orcid.org/0000-0001-9826-6314

Andrés Emilson Delgado Truque1 orcid.org/0000-0002-1307-096X

María Eugenia Casanova' ${ }^{1}$ orcid.org/0000-0002-5724-2938

\author{
1. Grupo Interinstitucional de Medicina Interna (GIMI 1), Universidad Libre Seccional Cali. Cali, Colombia \\ 2. Docente, Universidad Cooperativa de Colombia. Pasto, Colombia \\ 3. Docente, Universidad San Martín. Pasto, Colombia
}

\section{Resumen}

Introducción: La enfermedad de cambios mínimos es una causa rara de síndrome nefrótico en el adulto, y su relación con el hipotiroidismo es más rara aún. Se considera que esta patología renal responde favorablemente al manejo con glucocorticoides y tiene una baja frecuencia de resistencia. Su abordaje hoy en día es objeto de investigación. Objetivo: Describir una rara etiología de síndrome nefrótico en el adulto con presentación, tratamiento y desenlace infrecuentes. Presentación del caso: Paciente femenino de 53 años quien inicia con síndrome nefrótico por enfermedad de cambios mínimos cortico-resistente y su asociación con un hipotiroidismo descontrolado, quien requiere manejo con rituximab y control de la enfermedad endocrinológica asociada, como enfermedad de base. Conclusiones: En este caso clínico se muestra como las enfermedades citadas pueden coexistir y el tratamiento en conjunto es necesario. El establecimiento de nuevas terapias en la población adulta como el rituximab podría mostrar beneficio, como en este caso. Sin embargo, aún existe la necesidad de estudios de mayor evidencia que validen firmemente la efectividad de los diferentes tratamientos en este tipo de pacientes.

Palabras clave: Glomerulonefritis de cambios mínimos; síndrome nefrótico; hipotiroidismo. (Fuente: DeCS, Bireme).

\begin{abstract}
Introduction: Minimal change disease is a rare cause of nephrotic syndrome in adults, and its association with hypothyroidism is even more exceptional. This renal pathology is considered to respond favorably to glucocorticoid management and has a low resistance frequency. How to approach this disease is currently under investigation. Objective: To describe a rare etiology of adult nephrotic syndrome with unusual presentation, treatment, and outcome. Case presentation: A 53 years-old female patient who initially experienced nephrotic syndrome due to steroid-resistant minimal change disease, which was also associated with uncontrolled hypothyroidism. She required management with rituximab and control of the associated endocrinological disease, which was considered as the underlying disease. Conclusions: This clinical case shows (i) how the two aforementioned diseases can coexist and (ii) that a joint treatment is necessary. Establishing new therapies may be beneficial for adult populations, such as the benefits seen in this case with the use of rituximab. However, further studies are needed to strongly validate the effectiveness of the different treatments for these types of patients.
\end{abstract}

Keywords: Minimal change glomerulonephritis; nephrotic syndrome; hypothyroidism. (Source: DeCS, Bireme).

\footnotetext{
*Autor de correspondencia

José Leonel Zambrano Urbano

e-mail: jose.zambrano@hotmail.com
} 


\section{Introducción}

La nefropatía de cambios mínimos es la tercera causa del síndrome nefrótico en los adultos, a diferencia de la población pediátrica en donde es la etiología más prevalente(1); esta se caracteriza por grandes pérdidas de proteínas en la orina debido al daño glomerular a nivel de los podocitos como se ha documentado por microscopia electrónica(2).

Aunque ha sido ampliamente descrito y estudiado en la población pediátrica, existe una brecha en el conocimiento con relación al manejo en la población adulta, en especial en la variedad cortico-resistente que representa menos del $10 \%$ de los $\operatorname{casos}^{(3)}$, en donde se hace necesario la utilización de terapias adicionales como cilofosfamida, rituximab y tacrolimus con escasa evidencia en la literatura(4,5). Además, se desconocen los desenlaces a largo plazo y las posibles asociaciones con otras entidades como el hipotiroidismo(6). Se reporta el caso de una mujer de 53 años diagnosticada con síndrome nefrótico con presentación, tratamiento y desenlace infrecuentes.

\section{Presentación del caso}

Paciente femenina de 53 años, con antecedente de hipotiroidismo, en manejo con levotiroxina $112 \mathrm{mcg}$ al día y última TSH (Hormona Estimulante de Tiroides) hace 2 meses con valores normales $(2,35$ $\mathrm{mUI} / \mathrm{mL}$ ), quien consultó al servicio de urgencias por cuadro clínico de 2 días de evolución consistente en aparición de edema facial y miembros inferiores con predominio vespertino y empeoramiento progresivo, sin otros síntomas asociados. Al ingreso se encontró: Tensión Arterial: 150/100 mmHg y resto de signos vitales en rango normal, edema bipalpebral, facial, en hemiabdomen inferior y edema duro grado III de ambos miembros inferiores; se tomaron paraclínicos evidenciados en la Tabla 1, denotando hipertrigliceridemia, hipoalbuminemia y proteinuria en rango nefrótico (Figura 1), indicativo de síndrome nefrótico a descartar glomerulopatía primaria, resto de paraclínicos dentro de parámetros normales, pruebas de Hepatitis y VIH (Virus de inmunodeficiencia Humana) negativas.

Tabla 1. Parámetros de laboratorio tomados al ingreso Cali, Valle del Cauca 2020

\begin{tabular}{lrr}
\hline \multicolumn{1}{c}{ Parámetro } & Rango normal & Rango del paciente \\
\hline TSH & $0,35-4,94$ & $>100 \mu \mathrm{UI} / \mathrm{mL}$ \\
& $\mathrm{mUI} / \mathrm{mL}$ & \\
T4L & $0,9-2,3 \mathrm{ng} / \mathrm{mL}$ & $0,7 \mathrm{ng} / \mathrm{dL}$ \\
Albuminuria en & $<30 \mathrm{mg}$ & $500 \mathrm{mg}$ \\
orina espontánea & & \\
Triglicéridos & $40-170 \mathrm{mg} / \mathrm{dl}$ & $727 \mathrm{mg} / \mathrm{dL}$ \\
Colesterol total & $135-220 \mathrm{mg} / \mathrm{dl}$ & $737 \mathrm{mg} / \mathrm{dL}$, \\
Colesterol LDL & Menor de 150 & $539 \mathrm{mg} / \mathrm{dL}$ \\
& $\mathrm{mg} / \mathrm{dl}$ & $53 \mathrm{mg} / \mathrm{dL}$ \\
Colesterol HDL & Mayor a 35 & \\
& $\mathrm{mg} / \mathrm{dl}$ & $0,9 \mathrm{mg} / \mathrm{dL}$ \\
Creatinina & $0,7-1,5 \mathrm{mg} / \mathrm{dl}$ & $14814 \mathrm{mg} / \mathrm{día}$ \\
Proteinuria de 24 & & Proteínas:,+++ \\
horas & & Leucocitos: 68, \\
Uroanálisis & & cilindros granulosos \\
& & gruesos, \\
\hline
\end{tabular}

TSH: hormona estimulante de tiroides, T4L: tetrayodotiroxina libre, Colesterol LDL: colesteol baja densidad, Colesterol HDL: colesterol de alta densidad

Se complementó estudios con perfil inmunológico: ANAS, pANCAS, cANCAS, anti DNA (Anticuerpos antinucleares, anticitoplasma de neutrófilos con patrón de fluorescencia perinuclear y patrón de fluorescencia citoplásmico), complemento sérico C3, C4 y anticoagulante lúpico, anticuerpos anti beta 2 glicoproteína IgG e IgM, anti cardiolipina IgG e IgM negativos y ecografía renal sin alteraciones. Ante la asociación de hipotiroidismo y síndrome nefrótico se inició manejo con levotiroxina $200 \mathrm{mcg}$ día por vía oral e hidrocortisona $50 \mathrm{mg}$ cada 6 horas intravenosa.

Dado lo anterior, se decidió solicitar biopsia renal, la cual reportó "podocitopatia difusa de cambios mínimos, fibrosis intersticial y atrofia tubular mínima, arteriosclerosis leve a moderada": "enfermedad de cambios mínimos" (Figura 2). 


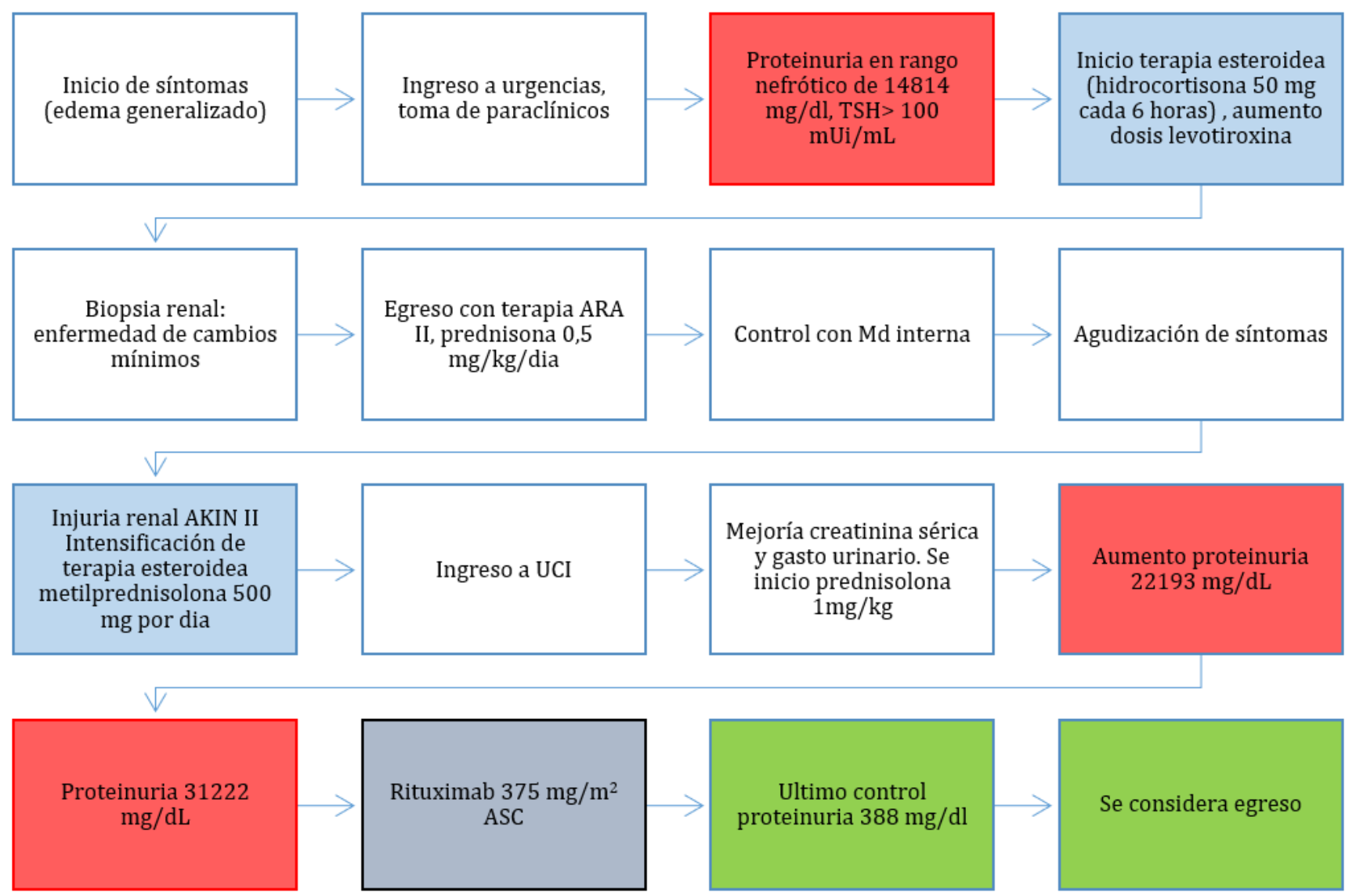

Figura 1. Línea de tiempo evolutiva del paciente.
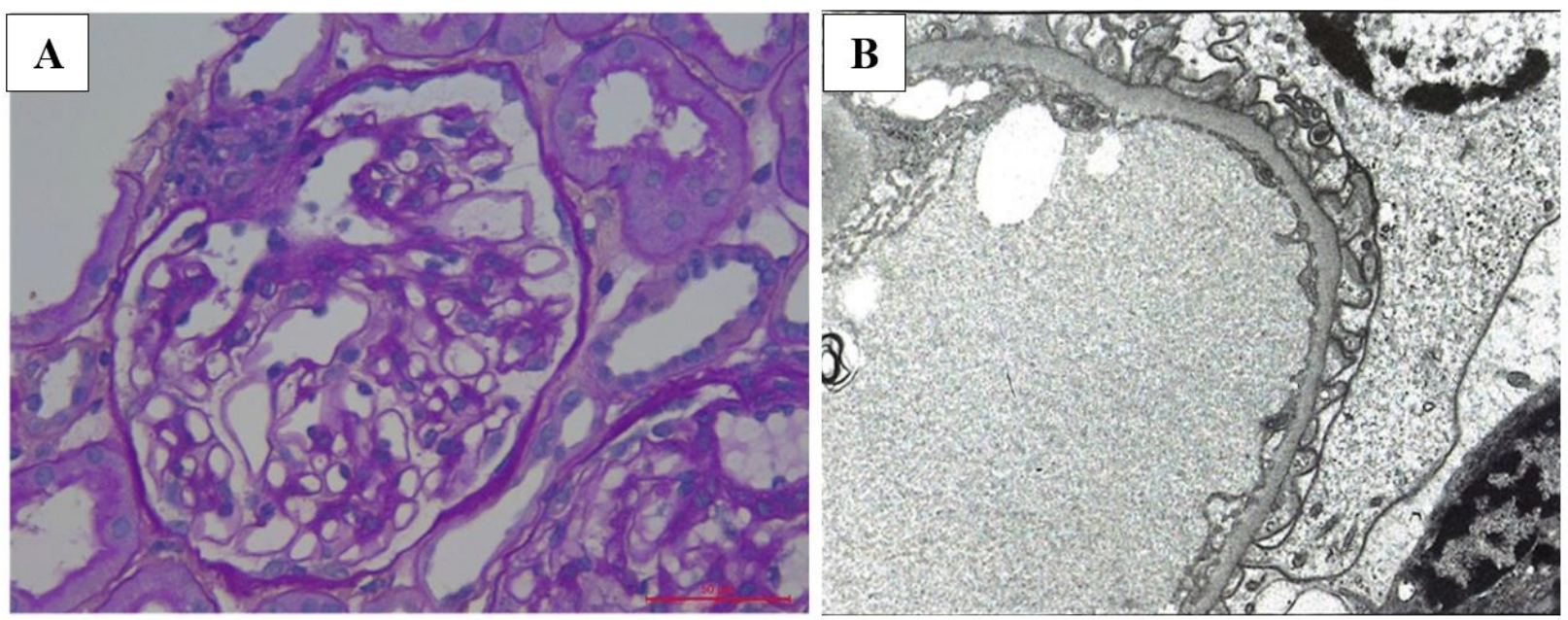

Figura 2. (A) Biopsia renal. Izquierda: microscopía óptica de la biopsia renal. La tinción de ácido periódico-Schiff mostró glomérulos sin cambios evidentes, fibrosis y atrofia tubular mínima. (B) Micrografía electrónica de biopsia renal, con borramiento difuso de los pedicelos. 
Por mejoría de edema generalizado y control de cifras tensionales, se dio egreso 6 días después con tratamiento antiproteinúrico con ARA II (valsartan $160 \mathrm{mg}$ cada 12 horas) y prednisolona a 0,5 $\mathrm{mg} / \mathrm{kg} /$ día. Se citó a control en 2 meses en donde no se evidenció cambios significativos clínicamente en los paraclínicos de control.

Aproximadamente después de 4 semanas, la paciente presentó reaparición y empeoramiento de edemas, así como aumento de aproximadamente $6 \mathrm{~kg}$ de su peso y dificultad respiratoria por congestión pulmonar, por lo que consultó nuevamente reportándose proteinuria de $17000 \mathrm{mg} /$ día, injuria renal aguda AKIN II, con elevación de creatinina sérica $(1,83 \mathrm{mg} / \mathrm{dL})$ y caída del gasto urinario $(0,2$ $\mathrm{mL} / \mathrm{Kg} / \mathrm{min}$ ), ingresando a UCI (Unidad de cuidados intensivos) donde se inició manejo con furosemida intravenosa en infusión a $5 \mathrm{mg}$ hora y espirinolactona a dosis 75mg día, infusión de albúmina, además, optimización de terapia esteroidea con pulsos de metilprednisolona $500 \mathrm{mg}$ IV cada 24 horas por 3 dosis. Una vez se completó el ciclo de esteroides intravenosos con evidencia de mejoría en cuanto a niveles séricos de creatinina y mejores tasas de diuresis, se cambió a terapia oral con prednisolona a dosis de $1 \mathrm{mg} / \mathrm{Kg}$ y se corroboró resultados de biopsia con el diagnóstico de enfermedad de cambios mínimos.

Sin embargo, en paraclínicos de control se evidenció aumento valores de proteinuria desde $22193 \mathrm{mg}$ /día al día 105 desde el inicio del cuadro, hasta 31222 mg/día en el día 112 (Figura 1), diagnosticándose de esta manera corticoresistencia a las 16 semanas del tratamiento esteroideo, razón por la que se decidió iniciar rituximab $375 \mathrm{mg} / \mathrm{m}^{2}$ ASC. Posteriormente, la paciente evidenció mejoría clínica, así como en paraclínicos de TSH y T4L dentro de la normalidad y proteínas en orina espontánea en disminución, con creatinina sérica de control normal $(0,9 \mathrm{mg} / \mathrm{dL})$ y proteinuria en orina de 24 horas de $388 \mathrm{mg} / \mathrm{dL}$ a los 7 días posteriores a la administración de rituximab, razón por la cual se consideró egreso (Figura 3).

Se continuó régimen de rituximab igual dosis por 4 ciclos y posibilidad de repetición si lo ameritara en un futuro, con disminución progresiva de dosis de corticoide a dosis mínima (10 mg cada día) para control de la enfermedad. Actualmente posterior a las 4 dosis, la paciente se encuentra asintomática 20 meses después sin necesidad de nuevos ciclos de anti CD20.

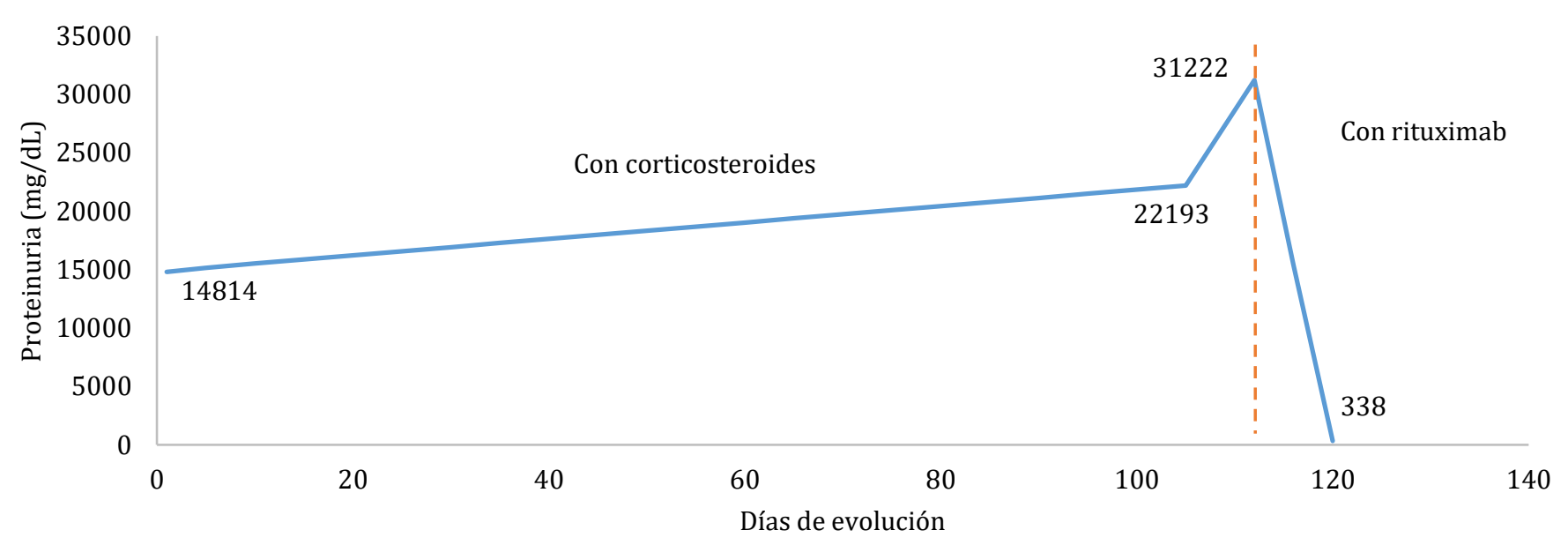

Figura 3. Evolución de la proteinuria $(\mathrm{mg} / \mathrm{dL})$ del paciente según los días de evolución y tratamiento

\section{Consideraciones éticas}

Este trabajo contó con el aval del Comité de Ética del Hospital San Miguel de Cali, Colombia para la publicación del caso y tuvo en cuenta las normas vigentes sobre investigación en seres humanos, contempladas en la Declaración de Helsinki de la
Asociación Médica Mundial y la Resolución 8430 de 1993 del Ministerio de Salud de Colombia. De igual manera se obtuvo el consentimiento informado de su tutora legal para la elaboración de historia clínica, recolección de datos e imágenes fotográficas y posterior publicación. 


\section{Discusión}

La enfermedad de cambios mínimos es una entidad poco estudiada en la población adulta dada la baja prevalencia de esta según el grupo etario(5). En este reporte de caso se presenta una paciente de 53 años, quien inició con síndrome nefrótico bajo el contexto de enfermedad por cambios mínimos asociada a hipotiroidismo descontrolado. La asociación entre ambas etiologías es rara, y la fisiopatología que entrama esta relación sigue en estudio, se ha descrito varias anormalidades que afectan la inmunidad celular y humoral en individuos afectados, en donde se destaca la activación de las células $\mathrm{T}$ y aumento de citocinas como el "factor permeabilizador" que median la injuria a las células podocitarias(7).

Se ha observado que los paciente con síndrome nefrótico, pueden perder hormonas de T4 por la orina, sin embargo, son capaces de responder a estas pérdidas aumentado la producción de esta hormona; a pesar de esto los pacientes que ya tienen hipotiroidismo de base $y$ vienen tomando tratamiento supletorio, no son capaces de responder a estas pérdidas, lo que empeora el hipotiroidismo, resultando como único tratamiento la resolución de la proteinuria(6,8).

Como segundo dato relevante en este caso, la enfermedad de cambios mínimos de la paciente se comportó como corticoresistente (definido como el tratamiento con esteroides optimo por dieciséis semanas sin respuesta satisfactoria), condición muy rara de comportamiento de esta enfermedad $(5,9,10)$. En el 2018 Fenton et al., publicaron un estudio de cohortes retrospectivo observacional analizando 78 pacientes adultos con enfermedad de cambios mínimos mostrando que muchos de los pacientes en terapia con esteroides a menudo requerían medicación adicional para el tratamiento de las recaídas utilizando medicamentos como el rituximab(9); sin embargo, se destaca la falta de estudios más extensos evaluando estas terapias. En esta paciente la intensificación de la terapia esteroidea no aportó beneficios clínicos significativos.

Diversos autores han enfocado esfuerzos a la estandarización terapéutica resaltando la duración de la misma, mostrando que los regímenes extendidos en duración de la terapia esteroidea (mayor de 2 meses) no aportaba beneficios terapéuticos significativos(11,12). Las causas fisiopatológicas de la corticoresistencia continúan en estudio; se ha registrado que los pacientes corticoresistentes expresan mayor cantidad de endotelina, molécula que promueve la alteración del citoesqueleto de actina en el podocito y aumenta la permeabilidad a la albúmina(13).

El tratamiento de una persona adulta bajo estas condiciones connota una mayor dificultad debido a la falta de estudios, en comparación con la edad pediátrica, demostrando que aún existe falta de claridad para establecer una pauta terapéutica estandarizada(12). Existen algunas investigaciones que demuestran la efectividad de esta terapia, como el estudio multicéntrico, doble ciego, a cargo de Lijima et al.(14), quienes demuestran la eficacia en la prevención de recaídas y la seguridad en el tratamiento en la población pediátrica, sin embargo, los estudios del rituximab en la edad adulta son escasos(9,15-17). Un estudio de cohortes retrospectivo, realizado por Takayuki Katsuno en 2018, evaluó la eficacia de rituximab a dosis de $375 \mathrm{mg} / \mathrm{m}^{2}$ en el tratamiento de síndrome nefrótico en adultos cuya etiología era la enfermedad de cambios mínimos, mostrando de igual manera la diminución de las recaídas, la dosis de inmusupresores y de esteroidea, este estudio contó con un bajo número de participantes (8)(15).

El mecanismo que explica la acción de este medicamento sigue en estudio, se ha descrito que regula la acción de los linfocitos $\mathrm{T}$, los factores de permeabilidad, puede iniciar la estabilización del citoesqueleto de los podocitos, y prevenir la apoptosis, a través de la interacción con el epitelio glomerular, además actuando como un anticuerpo monoclonal dirigido al antígeno CD20 de los linfocitos B, en el tratamiento del síndrome nefrótico idiopático, disminuyendo el grado de respuesta inflamatoria y condicionando un depleción de linfocitos $\mathrm{B}^{(14-16)}$.

Lo anterior proporciona un panorama favorable para próximas investigaciones con mayor valor epidemiológico como ensayos clínicos aleatorizados, dada la limitada literatura que se tiene en este escenario, además puede abrir el camino hacia otras terapias como el tacrolimus que igualmente se ha investigado y ha demostrado efectividad similar a la de los esteroides en el tratamiento(17,18). 


\section{Conclusiones}

Dada la rareza de la enfermedad de cambios mínimos y más aún su presentación cortico resistente. Este artículo muestra los resultados benéficos de la terapia con rituximab en una paciente con estas características, que, además, mejoró la exacerbación de su enfermedad de base. El estudio de estas patologías y su contextualización evidencian la necesidad de ampliar la información y fortalecer la evidencia que soporte la sospecha diagnóstica de esta entidad, al igual que el tratamiento en la población adulta.

Conflicto de intereses: Ninguno declarado por los autores.

\section{Referencias}

1. Ares Blanco S, Castillo López C, Villar Coloma E. Síndrome nefrótico primario: manejo desde atención primaria. FMC Form Médica Contin En Aten Primaria [Internet]. 2018 Dec [consultado 2021 Mar 13];25(10):581-4. DOI: 10.1016/j.fmc.2017.11.009.

2. National Kidney Foundation. Minimal Change Disease [Internet]. 2016 [consultado 2021 Mar 13]. Disponible en: https://www.kidney.org/atoz/content/minimal-changedisease

3. Patil MR, Divyaveer SS, Raychaudhary A, Trivedi M, Mahajan C, Sarkar D, et al. Tacrolimus as the first-line agent in adultonset minimal change disease: A randomized controlled study. Saudi J Kidney Dis Transplant. 2019 Feb;30(1):129-37. Disponible en: https://pubmed.ncbi.nlm.nih.gov/30804274

4. Vivarelli M, Massella L, Ruggiero B, Emma F. Minimal Change Disease. Clin J Am Soc Nephrol. 2017 Feb 7;12(2):332-45. DOI: 10.2215/CJN.05000516.

5. Iwabuchi Y, Miyabe Y, Makabe S, Nakano M, Manabe S, Karasawa K, et al. Comparison of the response of frequently relapsing steroid-dependent minimal change nephrotic syndrome to rituximab therapy between childhood-onset and adult-onset disease. Medicine [Internet]. 2018 Oct [consultado 2021 Mar 13]; 97(42):e12704. DOI: 10.1097/MD.0000000000012704.

6. Li L-Z, Hu Y, Ai S-L, Cheng L, Liu J, Morris E, et al. The relationship between thyroid dysfunction and nephrotic syndrome: a clinicopathological study. Sci Rep [Internet]. 2019 Apr 23;9(1):6421. DOI: 10.1038/s41598-019-42905-4.

7. Mathieson PW. Immune dysregulation in minimal change nephropathy. Nephrol Dial Transplant [Internet]. 2003 Aug;18(Suppl 6):vi26-9. DOI: 10.1093/ndt/gfg1066.

8. Park SJ, Shin JI. Complications of nephrotic syndrome. Korean J Pediatr [Internet]. 2011 Aug [consultado 2021 Nov 29];54(8):322-8. DOI: 10.3345/kjp.2011.54.8.322.

9. Fenton A, Smith SW, Hewins P. Adult minimal-change disease: observational data from a UK centre on patient characteristics, therapies, and outcomes. BMC Nephrol [Internet]. 2018 [consultado 2021 Mar 13];19(1):207. DOI: 10.1186/s12882-018-0999-X.

10. Nishi S, Ubara Y, Utsunomiya Y, Okada K, Obata Y, Kai H, et al. Evidence-based clinical practice guidelines for nephrotic syndrome 2014. Clin Exp Nephrol [Internet]. 2016 Jun [consultado 2021 Mar 13];20(3):342-70. DOI: 10.1007/s10157-015-1216-x.

11. Korbet SM, Whittier WL. Management of Adult Minimal Change Disease. Clin J Am Soc Nephrol [Internet]. 2019 Jun 7 [consultado 2021 Mar 13];14(6):911-3. DOI: 10.2215/CJN.01920219.

12. Bomback AS. Shortening the Duration of Corticosteroid Exposure in Minimal Change Disease: Can We Treat Adults Like Children? Am J Nephrol [Internet]. 2019 [consultado 2021 Mar 13];49(1):52-3. DOI: 10.1159/000495351.

13. Ahmed HM, Morgan DS, Doudar NA, Naguib MS. High Serum Endothelin-1 Level is Associated with Poor Response to Steroid Therapy in Childhood-Onset Nephrotic Syndrome. Saudi J Kidney Dis Transplant [Internet]. 2019 Aug;30(4):769-74. DOI: 10.4103/1319-2442.265451.

14. Iijima K, Sako M, Nozu K, Mori R, Tuchida N, Kamei K, et al. Rituximab for childhood-onset, complicated, frequently relapsing nephrotic syndrome or steroid-dependent nephrotic syndrome: a multicentre, double-blind, randomised, placebo-controlled trial. The Lancet [Internet]. 2014 Oct [consultado 2021 Mar 13];384(9950):1273-81. DOI: 10.1016/S0140-6736(14)60541-9.

15. Katsuno T, Masuda T, Saito S, Kato N, Ishimoto T, Kato S, et al. Therapeutic efficacy of rituximab for the management of adult-onset steroid-dependent nephrotic syndrome: a retrospective study. Clin Exp Nephrol [Internet]. 2019 Feb [consultado 2021 Mar 13];23(2):207-14. DOI: 10.1007/s10157-018-1630-y.

16. Waldman M, Crew RJ, Valeri A, Busch J, Stokes B, Markowitz $\mathrm{G}$, et al. Adult Minimal-Change Disease: Clinical Characteristics, Treatment, and Outcomes. Clin J Am Soc Nephrol [Internet]. 2007 May [consultado 2021 Mar 13];2(3):445-53. DOI: 10.2215/CJN.03531006.

17. Fornoni A, Sageshima J, Wei C, Merscher-Gomez S, Robier A$\mathrm{P}$, Jauregui AN, et al. Rituximab targets podocytes in recurrent focal segmental glomerulosclerosis. Sci Transl Med [Internet]. 2011 Jun 1 [consultado 2021 Mar 13];3(85):85ra46. DOI: 10.1126/scitranslmed.3002231.

18. Balwani MR, Bawankule CP, Pasari A, Tolani P, Vakil S, Yadav R. Minimal change disease and Kimura's disease responding to tacrolimus therapy. Saudi J Kidney Dis Transplant [Internet]. 2019 Jan 1 [consultado 2021 Mar 13];30(1):2547. DOI: $10.4103 / 1319-2442.252921$.

19. Ares Blanco S, Castillo López C, Villar Coloma E. Síndrome nefrótico primario: manejo desde atención primaria. FMC Form Médica Contin En Aten Primaria. 2018 Dec [consultado 2021 Mar 13];25(10):581-4. DOI: 10.1016/j.fmc.2017.11.009. 